
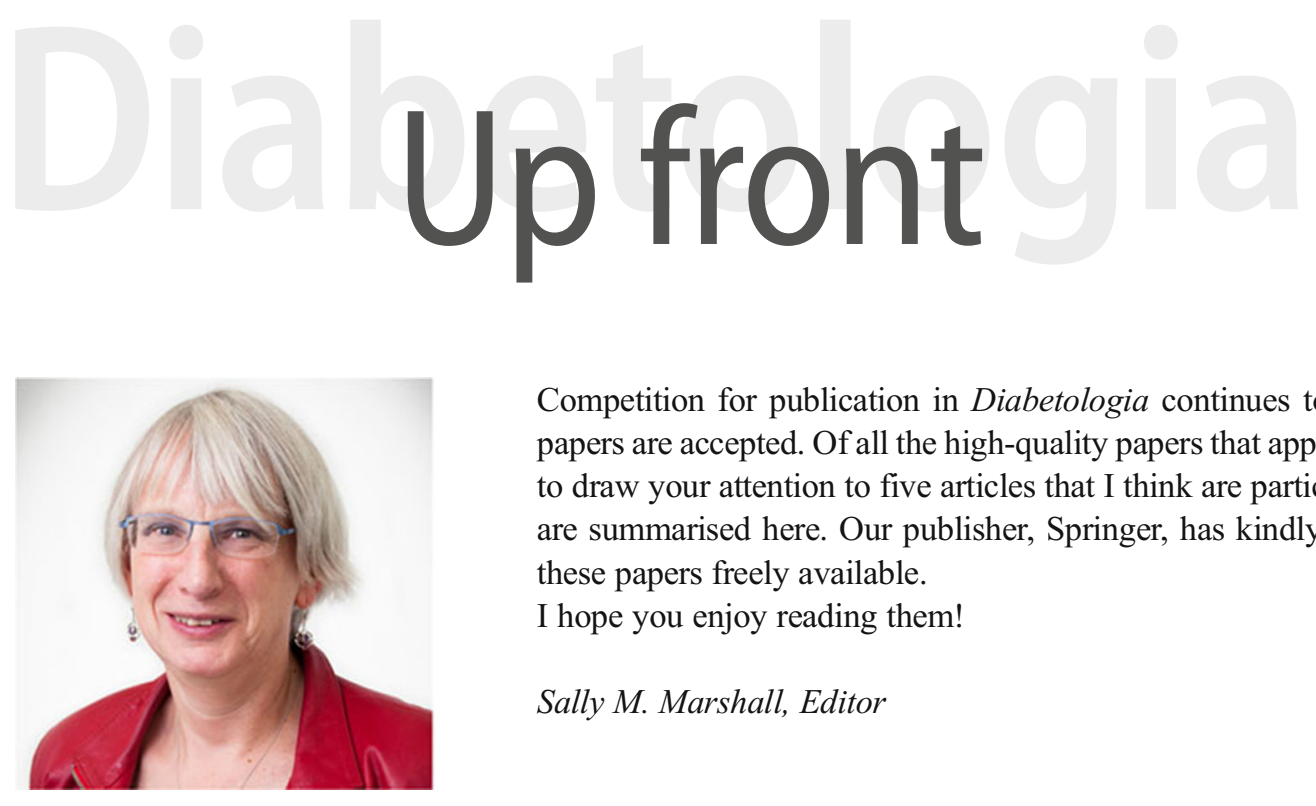

Competition for publication in Diabetologia continues to grow, and less than $20 \%$ of papers are accepted. Of all the high-quality papers that appear in this month's issue I want to draw your attention to five articles that I think are particularly interesting. The articles are summarised here. Our publisher, Springer, has kindly made the full text of each of these papers freely available.

I hope you enjoy reading them!

Sally M. Marshall, Editor

\section{Brain control of blood glucose levels: implications for the pathogenesis of type 2 diabetes}

Kimberly M. Alonge, David A. D’Alessio, Michael W. Schwartz

In this issue, Alonge and colleagues (https://doi.org/10. 1007/s00125-020-05293-3) present a framework for understanding the brain's role in glucose homeostasis. Their review outlines a crucial role for the brain in sensing blood glucose levels and they cite evidence in support of the concept that defects in this process are fundamental to the pathogenesis of type 2 diabetes. This framework also proposes that the close association between obesity and type 2 diabetes arises from a shared defect in overlapping and highly integrated brain neurocircuitry governing energy homeostasis and glucose homeostasis. The authors highlight the translational potential of this framework by summarising evidence that, in preclinical models of type 2 diabetes, blood glucose levels can be restored to normal in a sustained manner by therapies targeting the brain. They conclude that these considerations suggest that, unlike current non-surgical treatment options, strategies targeting the brain have the potential to induce sustained remission of type 2 diabetes.

(4) The figures from this review are available as a downloadable slideset.
Presumption of guilt for $\mathbf{T}$ cells in type 1 diabetes: lead culprits or partners in crime depending on age of onset?

Alexia Carré, Sarah J. Richardson, Etienne Larger, Roberto Mallone

It is increasingly recognised that autoimmune type 1 diabetes is a tale of two main characters that both play a role in disease pathogenesis: the T cell and the beta cell. On one hand, the presumption of innocence traditionally granted to beta cells requires revision, as recently reviewed in Diabetologia by Mallone and Eizirik (https://doi.org/10. 1007/s00125-020-05176-7). On the other hand, the presumption of guilt that $\mathrm{T}$ cells are often charged with may also need to be reconsidered. In this review, Carré et al (https://doi.org/10.1007/s00125-020-05298-y) evaluate genetic, histopathological, functional and clinical evidence to argue that T cells may behave as 'lead culprits' or 'partners in crime' in type 1 diabetes, depending on specific disease endotypes, mostly related to age. Increasing evidence points to the existence of a youngeronset endotype with more aggressive autoimmunity and an older-onset endotype with more vulnerable beta cells. Current challenges and opportunities for dissecting this disease heterogeneity are discussed.

(屯) The figures from this review are available as a downloadable slideset. 
Maternal blood glucose level and offspring glucose-insulin homeostasis: what is the role of offspring adiposity?

Ellen C. Francis, Dana Dabelea, Brandy M. Ringham, Katherine A. Sauder, Wei Perng

Maternal blood glucose status during pregnancy is associated with offspring adiposity and metabolic health; however, it is unclear whether offspring adiposity at birth and/or in childhood mediates the association between maternal glucose and offspring glucose-insulin homeostasis. In this issue, Francis et al (https://doi.org/10.1007/s00125-020-05294-2) report that higher maternal $\mathrm{HbA}_{1 \mathrm{c}}$ was associated with higher fasting glucose and lower insulin sensitivity in offspring. Further, this relationship was not mediated by offspring adiposity at birth, at age 4-7 years, or cumulatively across the two life stages. The authors suggest that the effect of maternal glucose on offspring glucose-insulin homeostasis may operate through alternate pathways, such as fetal programming of the adipoinsular axis or pancreatic beta cell development and function. This highlights the importance of the pregnancy period for prevention efforts aimed at improving early childhood metabolic health.

Paracrine regulation of somatostatin secretion by insulin and glucagon in mouse pancreatic islets

Berit Svendsen, Jens J. Holst

The alpha and beta cells of the islets of Langerhans secrete glucagon and insulin, which play an important role in regulating plasma glucose concentration, whilst the islet delta cells produce somatostatin, which is known to be able to inhibit the secretion of the other two hormones. In the islets, the three cell types are arranged in a complex pattern that, together with the capillary network, seems to be consistent with extensive paracrine interactions. For example, insulin is thought to directly inhibit glucagon secretion and recent research has revealed that local glucagon secretion is also essential for proteinstimulated insulin secretion. However, whether somatostatin secretion is regulated by paracrine signalling from glucagon and insulin is less clear. In this issue, Svendsen and Holst (https://doi.org/10.1007/s00125-020-05288-0) describe how somatostatin powerfully inhibits glucagon secretion and, conversely, how glucagon secretion provides a powerful local stimulus for somatostatin secretion, linking the two in a reciprocal feed-back circuit. Insulin did not directly affect secretion of somatostatin in mouse islets. The authors conclude that the results underline the importance of paracrine intra-islet regulation and suggests that a defect in one hormone will affect the secretion of other islet hormones. Thus, knowledge about islet somatostatin secretion appears to be essential for the understanding of integrated islet function.

Hypothalamic MC4R regulates glucose homeostasis through adrenaline-mediated control of glucose reabsorption via renal GLUT2 in mice

Leticia Maria de Souza Cordeiro, Arwa Elsheikh, Nagavardhini Devisetty, Donald A. Morgan, Steven N. Ebert, Kamal Rahmouni, Kavaljit H. Chhabra

Obesity and increased plasma insulin levels (insulin resistance) are major risk factors for type 2 diabetes in the general population. Yet, individuals with melanocortin 4 receptor (MC4R) deficiency either do not develop, or are at a relatively lower risk of developing, diabetes. To understand why MC4R deficiency prevents diabetes despite obesity and insulin resistance, de Souza Cordeiro et al (https://doi.org/10. $1007 / \mathrm{s} 00125-020-05289-\mathrm{z}$ ) investigated the function of this protein in glucose regulation using mouse models. The authors observed that lack of MC4R in mice increased glucosuria, thereby keeping blood glucose levels normal. The increase in urinary glucose excretion was due to reduced plasma adrenaline and expression of the glucose transporter GLUT2 in the kidneys. These findings demonstrate that elevated glucosuria may be one of the reasons why MC4R deficiency prevents diabetes. Based on the study findings, the authors propose that blocking kidney-specific GLUT2 has the potential to treat diabetes.

All text supplied by the authors. 